required mitral valve repair. Fortunately, the repair was entirely successful and the patient remains free of symptoms from the standpoint of both angina and mitral regurgitation.

\section{REFERENCES}

1. Kemp HG. Left ventricular function in patients with the anginal syndrome and normal coronary arteriograms. Am J Cardiol 1973;32:375-6.

2. Arbogast R, Bourassa MG. Myocardial function during atrial pacing in patients with angina pectoris and normal coronary arteriograms: comparison with patients having significant coronary artery disease. Am J Cardiol 1973;32:257-63.

3. Cannon RO, Watson RM, Rosing DR, Esptein SE. Angina caused by reduced vasodilator reserve of the small coronary arteries. J Am Coll Cardiol 1983;6:1359-73.

4. Cannon RO, Quyyumi AA, Schenke WH, Fananapazir L, Tucker EE, Gaughan AM, et al. Abnormal cardiac sensitivity in patients with chest pain and normal coronary arteries. J Am Coll Cardiol 1990;16:1359-66.

5. Lansing AM. Transmyocardial revascularization: mechanism of action with carbon dioxide and holmium-yttrium-aluminum-garnet lasers [letter]. J Thorac Cardiovasc Surg 1998;115: 1392.

\title{
ENDOGENOUS ENDOPHTHALMITIS RESULTING FROM STERNAL WOUND INFECTION AFTER CORONARY ARTERY BYPASS GRAFTING
}

\author{
Suresh K. Nahata, MD, Norman A. Saffra, MD, Mark H. Genovesi, MD, Mark W. Connolly, MD, and \\ Joseph N. Cunningham, Jr., MD, Brooklyn, N.Y.
}

Endogenous endophthalmitis is an uncommon complication after surgery, but its incidence is increasing because of increasing use of immunosuppressive drugs, spread of acquired immunodeficiency syndrome, and increased use of invasive procedures. The purpose of this case report is to alert cardiac surgeons about this complication, because a patient's vision can be saved only with an aggressive early intervention by an ophthalmologist. ${ }^{1}$

Case report. A 56-year-old man with non-insulin-dependent diabetes mellitus underwent uneventful double coronary artery bypass grafting in June 1996. A week after discharge from the hospital, the patient was readmitted with a sternal wound infection. He was empirically started on intravenous (IV) vancomycin and aztreonam (Azactam). Gram stain showed gram-positive cocci and culture grew Staphylococcus aureus. The patient was brought to the operating room and had wound debridement, internal fixation of the sternum, and pectoralis minor flaps for wound closure.

On the third postoperative day he started having pain in the right eye. The white blood cell count rose from 8,000 to $13,000 / \mathrm{mm}^{3}$ and a spiking fever developed. An urgent ophthalmology consultation was requested. On examina-

From the Department of Surgery, Maimonides Medical Center, 4802 Tenth Ave., Brooklyn, N.Y.

Received for publication Feb. 23, 1998; accepted for publication Feb. 27, 1998.

Address for reprints: Norman A. Saffra, MD, 921 49th St., Brooklyn, NY 11219.

J Thorac Cardiovasc Surg 1998;116:176-7

Copyright (c) 1998 by Mosby, Inc.

0022-5223/98 \$5.00+ $0 \quad \mathbf{1 2 / 5 4 / 9 0 1 9 4}$ tion, the right eye had hypopyon with a hazy vitreous and fundus and no red reflex. The vision was 20/70 in the right eye. The left eye had no abnormalities. A diagnosis of endogenous endophthalmitis was made and the patient was taken to the operating room on an emergency basis. The patient had an anterior chamber tap and pars plana vitrectomy with intravitreal injection of $1 \mathrm{mg}(0.1 \mathrm{ml})$ of vancomycin and $400 \mu \mathrm{g}(0.1 \mathrm{ml})$ of dexamethasone. He also received subconjunctival injections of vancomycin and dexamethasone, and both the anterior chamber and vitreous were cultured. In the postoperative period, the patient continued to receive IV vancomycin, and he was also started on a program of vancomycin, prednisolone, and cyclopentolate eye drops and the combination drug dexamethasone, neomycin, and polymyxin B (Maxitrol) eye ointment.

The patient continued to have spiking fever and the white blood cell count did not decrease. The sternotomy wound showed increased erythema, and we decided to return him to the operating room. Four days after the eye operation he had further debridement of the sternotomy wound with removal of screws and plates and with bilateral pectoralis major muscle flaps for wound closure. The cultures from the vitreous and anterior chamber showed no growth. At this time the patient's visual acuity and general condition continued to improve. The visual acuity $2 \frac{1}{2}$ weeks after the eye operation was $20 / 30$ in the right eye. The patient was discharged to his home 3 weeks after his admission with instructions to continue IV antibiotics for 6 more weeks.

Discussion. Endophthalmitis is an intraocular infection that can occur exogenously, as after ocular surgery or trauma (organisms introduced from outside), or endogenously (the primary focus of infection being within the 
body). Endogenous endophthalmitis accounts for less than $15 \%$ of all cases of endophthalmitis and usually occurs in patients with other predisposing conditions, such as diabetes mellitus. ${ }^{1}$ In addition, patients receiving immunosuppressive drugs, persons abusing IV drugs, and patients undergoing invasive procedures are at increased risk for endophthalmitis. The usual primary foci of infection in endogenous endophthalmitis are meningitis, endocarditis, urinary tract infection, and wound infection.

The organisms responsible for endogenous endophthalmitis are varied. Fungal infections account for more than $50 \%$ of cases, among which Candida albicans is the commonest. The maximum concentration of organisms in endogenous endophthalmitis is believed to be in and around ocular blood vessels, which accounts for the efficacy of IV antibiotics. ${ }^{2}$

The clinical presentation of patients with endogenous endophthalmitis is variable, depending on the virulence of the organism. Mild cases are characterized by white nodules on the iris, retina, and choroid, whereas severe cases are characterized by opacification of the media and high intraocular pressures. Diabetic patients with wound infections are particularly susceptible to endophthalmitis. ${ }^{3}$

The systemic evaluation of a patient with suspected endogenous endophthalmitis includes a thorough history and physical examination to locate the extraocular source of infection. Ophthalmic ultrasonography can be useful in diagnosis. Specimens from both the anterior chamber and vitreous should be cultured because of potentially low yield in culturing organisms in a partially treated condition. The differential diagnosis includes conjunctivitis, glaucoma, and uveitis, among others, and these need to be excluded.
Our patient had diabetes mellitus, underwent a major operation, and then had a wound infection. This placed him at increased risk for septicemia and endogenous endophthalmitis. The sternal wound was culture positive for Staphylococcus aureus, but no organisms were identified in the ocular cultures, perhaps because IV antibiotics were begun 4 days before the diagnosis of endogenous endophthalmitis. Organisms are identified in about two thirds of patients with clinically diagnosed endophthalmitis.

The treatment of the patient with endogenous endophthalmitis consists of systemic and intravitreal antibiotics as soon as the diagnosis is made. The role of vitrectomy is complex. Its purpose is to obtain a good culture specimen, to remove toxins and cells, and to create space for intravitreal antibiotics.

In conclusion, if a patient has eye pain or visual disturbance after a cardiac operation and if he or she has a known source of infection, the diagnosis of endophthalmitis should be considered.

\section{REFERENCES}

1. Okada AA, Johnson RP, Liles WC, D'Amico DJ, Baker AS. Endogenous bacterial endophthalmitis: report of a ten-year retrospective study. Ophthalmology 1994;101:832-8.

2. Endophthalmitis Vitrectomy Study Group. Results of the endophthalmitis vitrectomy study. Arch Ophthalmol 1995;113: 1479-96.

3. Phillips WB, Tasman WS. Postoperative endophthalmitis in association with diabetes mellitus. Ophthalmology 1994;101: $508-18$.

\section{ELECTROSURGERY WITH A FIBEROPTIC BRONCHOSCOPE AND A SNARE FOR ENDOTRACHEAL/ENDOBRONCHIAL TUMORS}

Motoyasu Sagawa, MD, Masami Sato, MD, Hiroto Takahashi, MD, Muneo Minowa, MD, Yasuki Saito, MD, and Shigefumi Fujimura, MD, Sendai, Japan

Endobronchial tumors sometimes cause stenosis of the major bronchi, which requires prompt palliation. A num-

From the Department of Thoracic Surgery, Institute of Development, Aging and Cancer, Tohoku University, Sendai, Japan.

Received for publication Jan. 2, 1998; accepted for publication Feb. 13, 1998

Address for reprints: Motoyasu Sagawa, MD, Department of Thoracic Surgery, Institute of Development, Aging and Cancer, Tohoku University, 4-1 Seiryo-machi, Aoba-ku, Sendai 980-77, Japan.

J Thorac Cardiovasc Surg 1998;116:177-9

Copyright (C) 1998 by Mosby, Inc.

0022-5223/98 $\$ 5.00+0 \quad \mathbf{1 2 / 5 4 / 8 9 6 6 7}$ ber of techniques for palliation have been reported, such as the neodymium-yttrium-aluminum-garnet (Nd-YAG) laser, ${ }^{1}$ endoscopic cryosurgery, ${ }^{1,2}$ and electrosurgery with a rigid bronchoscope. ${ }^{1,3}$ However, electrosurgery with a fiberoptic bronchoscope and a snare is not a widely known technique in the surgery of the respiratory tract. ${ }^{4,5}$ In this study, we evaluate the effectiveness and complications of the technique in nine cases of tracheobronchial tumors resected by this technique. The indications and limitations of the technique are also discussed.

Technique. Endobronchial electrosurgery was performed under local anesthesia with an electrosurgical unit (UES/UES-10, Olympus, Tokyo, Japan), a flexible fiberoptic bronchoscope (BF-1TR/BF-P30/BF-P240, Olympus), and a snare (SD-11L-1/SD-18C-1, Olympus). Oxy- 